\title{
Smoking and tuberculosis among silicotic patients
}

\author{
C.C. Leung*, W.W. Yew\#, W.S. Law*, C.M. Tam*, M. Leung*, Y.W. Chung*, \\ K.W. Cheung*, K.W. Chan ${ }^{\#}$ and F. Fu*
}

ABSTRACT: The aim of the present study was to investigate the relationship between smoking and tuberculosis (TB) among high-risk silicotic patients in Hong Kong.

A cohort of 435 silicotic patients tuberculin tested from 1995-2002 was prospectively followedup until the end of 2005. Baseline characteristics were analysed with respect to positive tuberculin reaction $(\geqslant 10 \mathrm{~mm})$ at baseline and subsequent development of TB.

Smoking, alcohol use and body mass index were independent predictors of positive tuberculin reaction at baseline in multiple logistic regression analysis. Total cigarette pack-yrs did not demonstrate any significant effect. The annual incidences of TB were 1,841, 2,294 and 4,181 per 100,000 for never-, ex- and current smokers, respectively. On Cox proportional hazard analysis, current smokers have a significantly higher risk of TB than other silicotic patients (adjusted hazard ratio (95\% confidence interval (CI)): 1.96 (1.14-3.35)) after controlling for age, alcohol use, tuberculin status, treatment for latent TB infection and other relevant background/disease factors. A significant dose-response relationship was also observed with the daily number of cigarettes currently smoked. Smoking cessation may reduce $32.4 \%$ (95\% Cl: 6.5-54.0) of the risk.

Smoking increases the risk of both tuberculosis infection and subsequent development of the disease among silicotic patients.

KEYWORDS: Cohort study, smoking, tuberculosis

I $\mathrm{n}$ recent years, there has been increasing evidence on the relationship between smoking and tuberculosis (TB). Smoking has been associated with latent TB infection (LTBI) [1-3], active TB disease [4-8] and TB-related death [912]. However, the underlying mechanisms of such associations remain largely elusive. As both conditions are strongly related to a multitude of socioeconomic factors, increased risk of exposure could explain, fully or in part, all the associations observed [13, 14]. The long latent period and relatively low incidence of active disease among immunocompetent subjects renders it rather difficult to dissect the complex relationships.

In Hong Kong, both smoking and TB are common conditions [15]. Silicosis is also the second most common statutorily notifiable occupational disease. Silicotic patients are regularly referred to the Pneumoconiosis Medical Board in the Tuberculosis and Chest Service of the Department of Health (Hong Kong, China) for compensation assessment. Confirmed silicotic patients are reassessed every 2 yrs for delineation of additional disability and also at death for contribution of the disease. During compensation assessment, detailed occupational and smoking histories are available. A very high risk of TB has been reported among these silicotic patients [5, 16]. Since 1995, tuberculin tests are also regularly offered to newly confirmed cases without previous history of TB. The availability of detailed assessment and tuberculin testing records in the present high-risk cohort allowed examination of the effect of smoking on TB prospectively and in greater detail.

\section{MATERIALS AND METHODS}

\section{Study subjects}

All silicotic patients without previous history of $\mathrm{TB}$, tuberculin skin testing or treatment for LTBI were offered a Mantoux test with purified protein derivative RT23 (1 unit from August 1, 1995 to June 30, 2000; 2 units from July 1, 2000 to December 31, 2002) as part of the regular service. Due to their existing small numbers, female subjects were excluded from the present study. The study was approved by the ethics committee of the Department of Health of Hong Kong.

\section{Study design}

A prospective cohort analysis was performed on the development of TB.

\section{AFFILIATIONS}

*Tuberculosis and Chest Service Centre for Health Protection, Department of Health, and

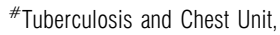
Grantham Hospital, Hong Kong, China.

\section{CORRESPONDENCE}

C.C. Leung

Pneumoconiosis Clinic

4/F 8 Chai Wan Road

Shaukeiwan

Hong Kong

China

Fax: 85229775940

E-mail: cc_leung@dh.gov.hk

Received:

October 162006

Accepted after revision:

December 042006

STATEMENT OF INTEREST

None declared. 


\section{Methods}

The following baseline information was collected at the time of tuberculin testing: date of tuberculin testing; name; age; identity card number; ethnicity; smoking history; alcohol use; bacilli Calmette-Guérin (BCG) scar; coexisting medical conditions; occupation; duration of dust exposure; and disease indices according to the International Labour Organisation classification (profusion/size/shape of lung nodules, progressive massive fibrosis). A never-smoker was defined as an individual who had never smoked as many as 1 cigarette $\cdot$ day $^{-1}$ or equivalent for the duration of $1 \mathrm{yr}$. An ever-smoker was defined as an individual who had smoked $\geqslant 1$ cigarette $\cdot$ day $^{-1}$ for $\geqslant 1$ yr. An ex-smoker was defined as an ever-smoker who had stopped smoking for $\geqslant 1 \mathrm{yr}$ prior to study commencement. A current smoker was defined as an ever-smoker who was still smoking within the previous $1 \mathrm{yr}$. A regular drinker was defined as an individual who drank alcohol regularly $\left(\geqslant 4\right.$ days $\cdot$ week $\left.^{-1}\right)$ at the time of tuberculin testing. The cohort was followed-up prospectively in the Pneumoconiosis Clinic (Hong Kong) or other chest clinics of the Tuberculosis and Chest Service until December 31, 2005. The cohort was also cross-matched with the statutory TB notification registry and death registry of Hong Kong for further case finding and ascertainment of vital status. The period of follow-up was defined as the period from enrolment to the date of notification of TB, death or December 31, 2005, whichever was earlier. Date of TB notification, bacteriological status, form of TB and previous TB history were retrieved from the notification registry. The diagnosis and clinical information of all identified TB cases were verified with the medical records retrieved from the Pneumoconiosis Clinic and other relevant sources. An active case of TB was defined as disease proven by isolation of Mycobacterium tuberculosis or, in the absence of bacteriological confirmation, disease diagnosed on clinical, radiological and/ or histological grounds together with an appropriate response to treatment.

\section{Analysis}

The effect of background and disease characteristics on LTBI (defined as a tuberculin reaction $\geqslant 10 \mathrm{~mm}$ ) on the baseline was first analysed in a cross-sectional analysis, followed by a prospective analysis with respect to the development of active TB disease and culture-proven TB. For univariate analysis, Chi-squared or Fisher exact tests were used for categorical variables and ANOVA for numerical variables as appropriate. Multiple logistic regression analysis and Cox proportional hazards analysis were then employed in the cross-sectional and prospective analysis, respectively, to control for all the confounding background/disease variables. A two-tailed pvalue $<0.05$ was taken as statistically significant. The risk of TB attributable to smoking was derived by applying the adjusted hazard ratio to the Levin formula [17]:

$$
\text { Attributable risk }=\mathrm{p} \times(\mathrm{r}-1) /[\mathrm{p} \times(\mathrm{r}-1)+1]
$$

where $\mathrm{p}$ is the proportion of the population exposed and $\mathrm{r}$ is the relative risk of disease for the exposed group.

\section{RESULTS}

A total of 441 silicotic patients were tuberculin tested from August 1, 1995 to December 31, 2002. Six female patients were

\begin{tabular}{|c|c|c|c|c|}
\hline $\begin{array}{l}\text { TABLE 1 } \begin{array}{l}\text { Backgrour } \\
\text { patients a }\end{array}\end{array}$ & $\begin{array}{l}\text { ר/clinical } \\
\text { t tuberculi }\end{array}$ & $\begin{array}{l}\text { haracteris } \\
\text { testing }\end{array}$ & cs of silic & otic \\
\hline \multirow[t]{2}{*}{ Variable } & \multicolumn{2}{|c|}{ Tuberculin reaction } & \multirow[t]{2}{*}{ Overall } & \multirow[t]{2}{*}{ p-value } \\
\hline & $<10 \mathrm{~mm}$ & $\geqslant 10 \mathrm{~mm}$ & & \\
\hline Subjects n & 118 & 317 & 435 & \\
\hline Age yrs & $58.4 \pm 9.9$ & $57.3 \pm 9.9$ & $57.6 \pm 9.9$ & 0.315 \\
\hline Smoking status & & & & 0.011 \\
\hline Never-smoker & 16.1 & 9.1 & 11.0 & \\
\hline Ex-smoker & 44.9 & 36.9 & 39.1 & \\
\hline Current smoker & 39.0 & 53.9 & 49.9 & \\
\hline Current cigarette $\cdot$ day $^{-1}$ & $3.9 \pm 8.3$ & $4.6 \pm 6.8$ & $4.4 \pm 7.3$ & 0.360 \\
\hline Cigarette pack-yrs & $24.0 \pm 20.1$ & $22.7 \pm 21.5$ & $23.1 \pm 21.1$ & 0.543 \\
\hline Regular alcohol use & 5.1 & 13.6 & 11.3 & 0.013 \\
\hline BCG scar & 1.7 & 3.8 & 3.2 & $0.369^{\#}$ \\
\hline BMI & $23.1 \pm 3.4$ & $23.8 \pm 3.3$ & $23.6 \pm 3.3$ & 0.079 \\
\hline With other comorbidities & 40.7 & 40.1 & 40.2 & 0.907 \\
\hline Principal job & & & & 0.104 \\
\hline Underground driller ${ }^{+}$ & 36.4 & 46.4 & 43.7 & \\
\hline Surface driller ${ }^{\S}$ & 31.4 & 32.2 & 32.0 & \\
\hline Fine silica ${ }^{f}$ & 5.1 & 3.2 & 3.7 & \\
\hline Other jobs ${ }^{\# \#}$ & 27.1 & 18.3 & 20.7 & \\
\hline Exposure to dust yrs & $24.2 \pm 9.4$ & $24.2 \pm 8.3$ & $24.2 \pm 8.6$ & 0.957 \\
\hline Profusion of nodules & & & & 0.066 \\
\hline Category 1 & 66.9 & 65.9 & 66.2 & \\
\hline Category 2 & 27.1 & 32.2 & 30.8 & \\
\hline Category 3 & 5.9 & 1.9 & 3.0 & \\
\hline Size of nodules $\mathrm{mm}$ & & & & 0.700 \\
\hline$<1.5$ & 28.0 & 32.2 & 31.0 & \\
\hline $1.5-3$ & 61.0 & 57.4 & 58.4 & \\
\hline $3-10$ & 11.0 & 10.4 & 10.6 & \\
\hline Regular shape of nodules & 81.4 & 82.3 & 82.1 & 0.878 \\
\hline $\begin{array}{l}\text { Progressive massive } \\
\text { fibrosis }\end{array}$ & 18.6 & 17.7 & 17.9 & 0.813 \\
\hline
\end{tabular}

Data are presented as \% or mean $\pm S D$, unless otherwise stated. BCG: bacille Calmette-Guérin; BMI: body mass index. " : Fisher exact, two-tailed p-value; ": hypertension, diabetes mellitus and other heart, cerebrovascular, lung, gastrointestinal and renal diseases; ${ }^{+}$: caisson workers/tunnel workers/miners; s: surface drillers/stone-splitters/drillers in the construction trade or quarries; $f$ :

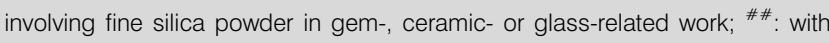
medium or low silica dust exposure.

excluded, leaving 435 male silicotic patients for analysis. All recruited subjects were ethnically Chinese.

Table 1 summarises the baseline characteristics of all recruited subjects as stratified by tuberculin status. Smoking was significantly associated with a positive tuberculin reaction $(\geqslant 10 \mathrm{~mm})$. Among the ex-smokers in the cohort, there was no significant difference in the tuberculin-positive rates $(66.2$ versus $70.5 \%, p=0.554$ ) between those 65 patients having given up smoking for $\geqslant 10 \mathrm{yrs}$ and the other 105 patients having given up for $<10$ yrs. Variables associated with a positive tuberculin response $(\geqslant 10 \mathrm{~mm})$ at a $\mathrm{p}$-value $<0.1$ were entered into multiple logistic regression and the results are summarised in table 2. Only smoking status, alcohol use and body mass index (BMI) remained independent predictors of a positive tuberculin reaction at baseline. Profusion of nodules 


\begin{tabular}{llc} 
TABLE 2 & $\begin{array}{l}\text { Predictors of positive tuberculin reaction }(\geqslant 10 \\
\text { mm) in multiple logistic regression analysis }\end{array}$ \\
Variable & OR $\mathbf{( 9 5 \% ~} \mathbf{~ l )}$ & p-value \\
\hline Smoking status & & \\
$\quad$ Never-smoker & $1.00($ Ref. $)$ & \\
Ex-smoker & $1.57(0.80-3.08)$ & \\
$\quad$ Current smoker & $2.72(1.37-5.40)$ & \\
Regular alcohol use & $2.72(1.11-6.62)$ & 0.028 \\
BMI per unit change & $1.08(1.01-1.15)$ & 0.032 \\
Profusion of nodules per & $0.96(0.64-1.41)$ & 0.826 \\
$\quad$ grade & & \\
\hline
\end{tabular}

OR: odds ratio; Cl: confidence interval; Ref.: reference; BMI: body mass index Only variables associated with positive tuberculin reaction at $p<0.10$ were entered into multiple logistic regression. Hosmer and Lemeshow test, Chisquared $=10.71, p=0.219$. Area under receiver operating characteristic curve: $64.6 \%(58.8-70.4)$

failed to reach statistical significance. Of the 317 patients with positive tuberculin reaction $\geqslant 10 \mathrm{~mm}$, only $101(31.9 \%)$ accepted treatment for LTBI; 61 were administered 6 months of daily isoniazid and 40 were administered 2 months of daily rifampicin and pyrazinamide.

The mean duration of follow-up from the day of enrolment to development of TB, death or December 31, 2005 was $1,908 \pm 847$ days. A total of 78 new TB cases were identified during the period of follow-up, with 75 cases from the regular clinical follow-up and three further cases from the TB notification registry. Two cases were found to have nontuberculous mycobacterial infection and three other bacteriologically negative cases were excluded due to absence of appropriate response after anti-TB treatment. The remaining 73 cases meeting the case definition for active TB were diagnosed at a mean duration of $1,273 \pm 874$ days from the time of tuberculin testing. In total, $49(67.1 \%)$ of them were confirmed by successful isolation of $M$. tuberculosis in culture. A total of 63 cases $(86.3 \%)$ had pulmonary involvement alone, two cases $(2.7 \%)$ presented extrapulmonary involvement alone and 8 cases $(11.0 \%)$ had both. None of the 66 TB patients consenting to voluntary HIV testing was found to be HIVinfected. A total of 54 deaths $(12.4 \%)$ occurred among the cohort, at a mean duration of $1,201 \pm 843$ days from the time of tuberculin testing. A total of 14 patients died from bronchogenic carcinomas, 11 from other malignancies, 16 from other respiratory diseases or complications, nine from cardiovascular diseases and four from miscellaneous conditions. Nine of the deaths occurred at varying intervals after the development of $\mathrm{TB}$, but none of them died directly as a result of the disease.

The annual incidences of active TB and culture-confirmed TB among different smoking categories are summarised in table 3 . For ex-smokers, there was no significant difference between individuals who had given up for $\geqslant 10$ yrs and those who had given up more recently in risk of active TB $(2,515$ per 100,000 versus 1,927 per 100,$000 ; p=0.588$ ) and culture-confirmed $\mathrm{TB}$ $(1,605$ per 100,000 versus 1,548 per 100,$000 ; \mathrm{p}=0.949)$. Current smokers were at a significantly higher risk of both active TB and culture-confirmed TB than those not currently smoking (never- and ex-smokers combined) and the differences persisted after all regular alcohol drinkers were excluded or after stratification by tuberculin status and treatment for LTBI (table 4).

The effects of different smoking-related variables on the risk of active TB disease and culture-confirmed TB in Cox proportional hazard analysis are summarised in table 5. Current smokers were at an increased risk for development of TB after adjustment by age, alcohol use, tuberculin status, treatment for LTBI and other relevant background/disease factors. Figures 1 and 2 demonstrate the higher risks of active TB and cultureconfirmed TB with increasing number of cigarettes smoked per day at the baseline, respectively. By applying the Levin formula, smoking at the time of tuberculin testing accounted for $32.4 \%$ (95\% confidence interval $6.5-54.0)$ and $36.0 \%$ (5.660.4 ) of the risks of active TB and culture-confirmed TB among the cohort, respectively.

\section{DISCUSSION}

With the potential ethical concerns in conducting a randomised controlled experiment on human subjects, a prospective cohort analysis is probably the best option to address the relationship between smoking and TB. Even in high incidence areas, the annual rate of $\mathrm{TB}$ among immunocompetent individuals is usually just in the order of one in 1,000 Therefore, it is necessary to follow-up a very large cohort to get sufficient cases for analysis, and with this, critical details about potential confounders are often incomplete or missing. The present cohort involved a relatively homogeneous group of male silicotic patients of Chinese ethnicity who were at a

TABLE 3 Annual rates of active tuberculosis (TB) and culture-confirmed TB by smoking status among the whole cohort

\begin{tabular}{|c|c|c|c|c|c|c|c|}
\hline \multirow[t]{2}{*}{ Smoking status } & \multirow[t]{2}{*}{ Subjects $n$} & \multicolumn{3}{|c|}{ Active TB } & \multicolumn{3}{|c|}{ Culture-confirmed TB } \\
\hline & & Cases n & Rate $^{\#}(95 \% \mathrm{Cl})$ & p-value & Cases n & Rate $^{\#}(95 \% \mathrm{Cl})$ & p-value \\
\hline Never-smoker & 48 & 5 & 1841 (598-4297) & & 3 & 1105 (228-3229) & \\
\hline Ex-smoker & 170 & 19 & 2294 (1381-3582) & 0.028 & 13 & 1570 (836-2684) & 0.079 \\
\hline Current smoker & 217 & 49 & $4181(3093-5527)$ & & 33 & $2816(1938-3954)$ & \\
\hline Total & 435 & 73 & $3213(2519-4040)$ & & 49 & 2157 (1596-2851) & \\
\hline
\end{tabular}

Cl: confidence interval. ${ }^{*}$ : calculated over a population of 100,000 . 


\begin{tabular}{llc} 
TABLE 4 & $\begin{array}{l}\text { Relative risks of active tuberculosis (TB) and } \\
\text { culture-confirmed TB between current smokers } \\
\text { and other silicotic patients }\end{array}$ \\
\cline { 2 - 3 } $\begin{array}{l}\text { Current smokers versus } \\
\text { other* }\end{array}$ & \multicolumn{2}{c}{ RR (95\% Cl) } \\
\cline { 2 - 3 } & Active TB & Culture-confirmed TB \\
\hline $\begin{array}{l}\text { Crude rates } \\
\text { Excluding regular drinkers } \\
\text { Stratified by TT/treatment }\end{array}$ & $1.96(1.18-3.12)$ & $1.94(1.07-3.52)$ \\
\hline
\end{tabular}

RR: relative risk; $\mathrm{Cl}$ : confidence interval; $\mathrm{TT}$ : tuberculin status. ${ }^{*}$ : includes both ex-smokers and never-smokers; "': Mantel-Haenszel analysis after stratification into three groups: tuberculin-negative, tuberculin-positive with latent TB infection treatment and tuberculin-positive without treatment.

very high risk for developing TB ( $3 \%$ per annum, as shown in table 3), and for whom detailed smoking history, tuberculin testing results and other clinical information were available under regular two-yearly compensation assessment. Therefore, it offers a relatively unique opportunity to examine the relationships between smoking, LTBI and TB.

In the present study, there was a high prevalence of LTBI as measured by a tuberculin reaction $\geqslant 10 \mathrm{~mm}$ (table 1 ), similar to what was previously reported among the silicotic patients in Hong Kong [5, 16, 18]. Both current and ex-smokers were associated with LTBI (table 1) and such association was independent of alcohol intake, BMI and other background variables (table 2). Never-smokers, ex-smokers and current smokers were at different risks of TB (table 3). Current smoking was consistently associated with higher rates of both active TB and culture-confirmed TB, even after exclusion of regular alcohol use or stratification by tuberculin status/ treatment of LTBI (table 4) and controlling for all relevant confounding variables (table 5). The time elapsed after giving up did not appear to affect the TB risk. A clear dose-response effect was also observed in active TB and culture-confirmed TB with the number of cigarettes smoked per day, again after controlling for all relevant confounders (table 5, fig. 1). Past cigarette pack-yrs showed only a suggestive but insignificant effect on the development of TB (table 5). The independent and dose-response relationship between current smoking and the subsequent development of $\mathrm{TB}$ disease is consistent with the authors' previous observations in another prospective cohort study [8]. In contrast with that study, none of the subjects had past history of TB and much more information was available regarding cigarette pack-yrs, time of giving up, tuberculin status and treatment history for LTBI.

With the closer examination allowed in the present study, there is further support of a causal link between smoking and TB. There is an increased vulnerability of developing disease, in addition to an increased risk of acquiring infection. For many years smoking has been associated with changes in pulmonary macrophages [19] and lymphocytes [20]. Pulmonary and upper zone predominance of TB lesions in smokers has been reported in a previous study [7]. More recent studies suggest that nicotine [21], similar to vagus efferent discharge [22], inhibits the release of tumour necrosis factor, which plays a key role in the cellular defence against TB [23]. The overall evidences are therefore in line with a direct airborne insult promoting the risk of disease development.

Tuberculin test was used to measure the LTBI because the specific interferon-release assays were not available locally at the time of the study [24]. As BCG vaccination was only introduced into Hong Kong in the 1950s [25] and the vast majority of the present cohort was born well before that time, BCG vaccination is unlikely to affect the interpretation of the test excessively. Indeed, only $3.2 \%$ of the present cohort showed a BCG vaccination scar and there was no significant association between a BCG scar and a positive tuberculin reaction.

The present study focused on a homogenous group of ethnically Chinese male patients with silicosis. It would be

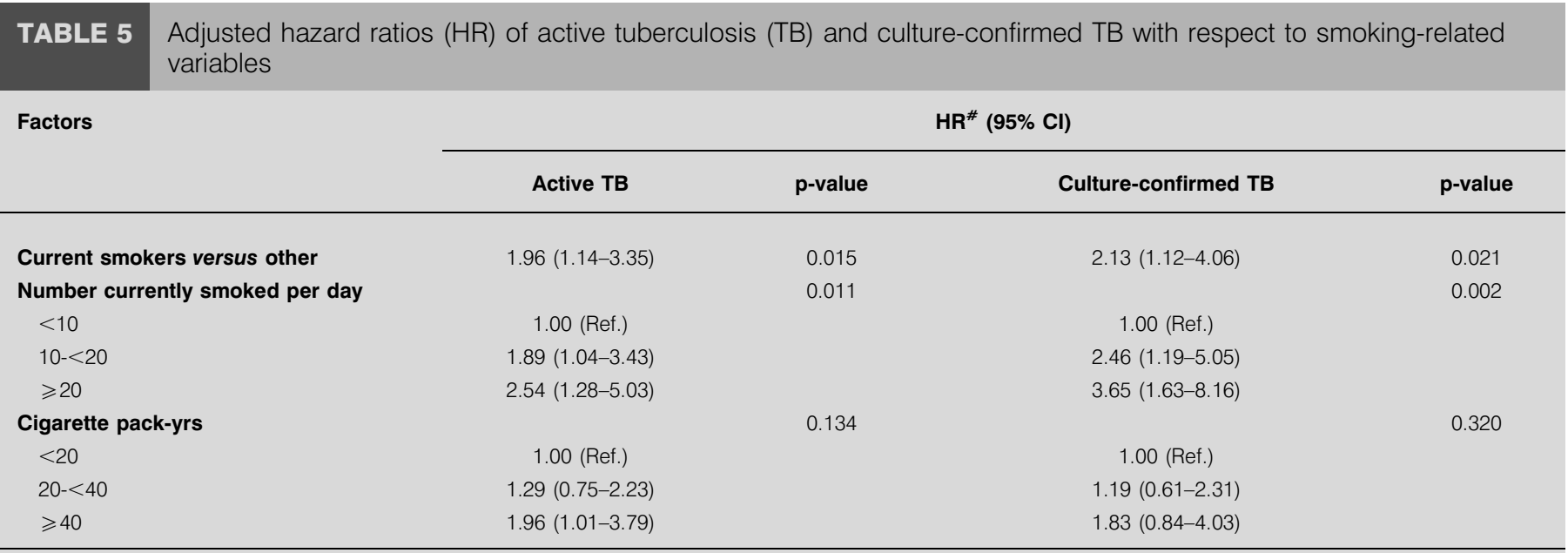

Cl: confidence interval; Ref.: reference. ${ }^{\#}$ : adjusted for age, past/current regular alcohol use, body mass index, presence of other comorbidities, bacille Calmette-Guérin scar, tuberculin status/treatment of latent TB infection, principal job type, duration of silica dust exposure, profusion, size and shape of lung nodules, and progressive massive fibrosis. 


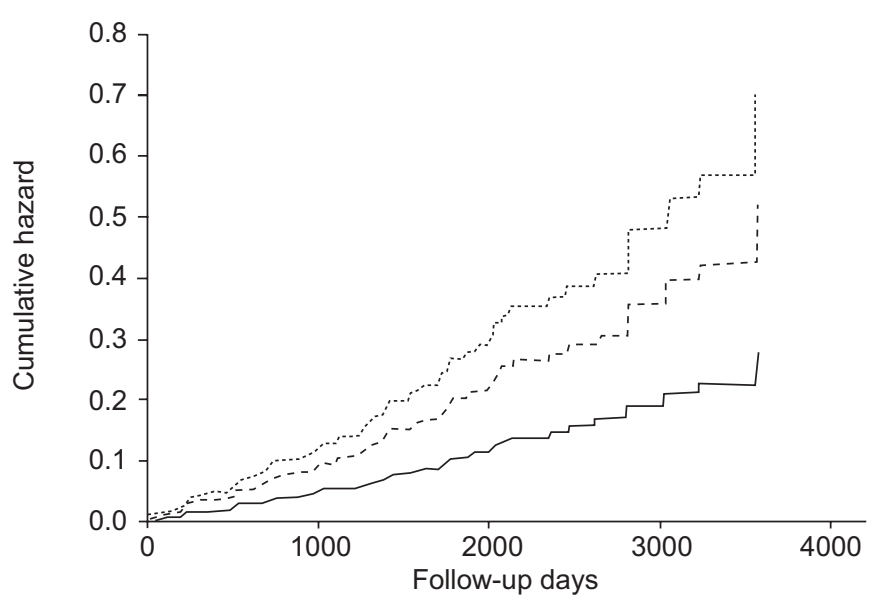

FIGURE 1. Risk of active tuberculosis among silicotic patients by number of cigarettes currently smoked per day after adjustment of other confounding variables, as listed in table $5 . \ldots$ _ $<10$ cigarettes.day ${ }^{-1} ;$-----: 10-19 cigarettes. day ${ }^{-1}$; .......: $\geqslant 20$ cigarettes.day ${ }^{-1}$. Overall model: $-2 \log$ likelihood 770.0 , Chi-squared 44.74; $\mathrm{p}<0.001$

ideal if it were indeed possible to examine a cohort involving all age groups in the general population. However, tuberculin test data are seldom available among other groups and their relatively low disease incidences pose a genuine difficulty in showing a difference between different smoking categories using active disease as an end-point. Therefore, the current cohort provides a possible window to examine the intricate relationship between smoking, LTBI and active TB. While there might be genuine concern that the findings might not necessarily be transferable to a general population, the higher risk of active TB in current smokers, as observed, is consistent with the findings in a similar study among a larger cohort of elderly subjects within the general community [8].

Incomplete case ascertainment is a major concern for prospective cohort analysis. As the cohort was under regular follow-up for compensation purposes and attempts have also been made to screen for missed cases through the territory-wide $\mathrm{TB}$ notification registry, the number of such cases should be low. In any case, incomplete case ascertainment, unless highly selective, is unlikely to significantly affect the internal comparison among different smoking categories. The vital status of patients missing follow-up was also ascertained through cross-matching with the territory-wide death registry. A relatively long period of cessation for $1 \mathrm{yr}$ was employed to distinguish between current and ex-smokers and this would have minimised the short-term fluctuations associated with abortive smoking cessation attempts. The diagnosis of TB could have been complicated by the progression of the underlying silicotic process. As current smokers were more likely to experience symptoms, this might increase the possibility of over-diagnosis of bacteriologically negative TB. However, the association persisted even if only culture-proven cases were considered.

Although alcohol use is a well-known confounder [26, 27], its prevalence was relatively low in the present cohort. Smoking showed independent effect on LTBI and development of TB, even after controlling for the effect of alcohol (tables 2, 4 and 5).

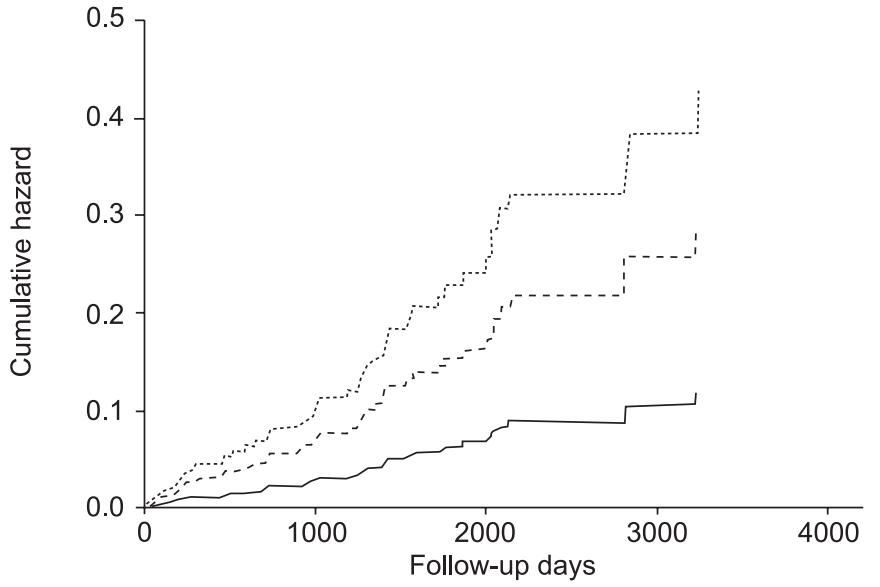

FIGURE 2. Risk of culture-confirmed tuberculosis among silicotic patients by number of cigarettes currently smoked per day after adjustment of other

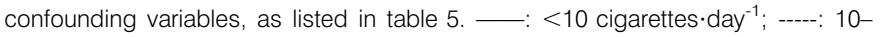
19 cigarettes $\cdot$ day $^{-1} ; \cdots \ldots .: 20$ cigarettes $\cdot$ day $^{-1}$. Overall model: $-2 l o g$ likelihood 520.1, Chi-squared 41.02; $p=0.001$.

In the absence of detailed local data on dust exposure levels for various jobs, only job type and duration of exposure were used as proxy measures of dust exposure and disease indices were also taken into account in the analysis. The prevalence of LTBI was not associated with specific job types (table 1) and the increased risk of current smokers persisted after adjustment of all relevant background variables and disease indices (table 5).

In the present study, one-third of the risk of tuberculosis disease was attributable to current smoking, partly reflecting the high smoking prevalence in the current cohort. Tuberculosis is an important cause of morbidity and mortality among silicotic patients [5] and both smoking and tuberculosis are independently associated with lung function impairment [28]. Smoking cessation is, therefore, expected to have major impact on the quality of life for patients suffering from this largely irreversible disease. Ageing of the population is also increasingly observed in developing countries, many of which still have a very high tuberculosis burden. In Hong Kong, $\sim 40 \%$ of tuberculosis patients are $\geqslant 65 \mathrm{yr}$ of age and males of $>80$ yrs of age have an eight-fold higher tuberculosis risk than the population average [15]. The interaction of smoking with the huge latently infected pool in an ageing population is therefore a serious concern. As endogenous reactivation accounted for $>70 \%$ of the tuberculosis risk in Hong Kong [29], the use of directly observed treatment, short course alone, is unlikely to be able to bring down the disease incidence rapidly. With the high male and rising female smoking prevalence in many parts of Asia, it would appear rational to include smoking cessation as one of the armaments for tuberculosis control.

\section{ACKNOWLEDGEMENTS}

The authors would like to express their sincere thanks to the medical and nursing staff of the Pneumoconiosis Clinic (Hong Kong, China) for assistance in data collection. 


\section{REFERENCES}

1 Anderson RH, Sy FS, Thompson S, Addy C. Cigarette smoking and tuberculin skin test conversion among incarcerated adults. Am J Prev Med 1997; 13: 175-181.

2 Solsona J, Cayla JA, Nadal J, et al. Screening for tuberculosis upon admission to shelters and free-meal services. Eur J Epidemiol 2001; 17: 123-128.

3 Plant AJ, Watkins RE, Gushulak B, et al. Predictors of tuberculin reactivity among prospective Vietnamese migrants: the effect of smoking. Epidemiol Infect 2002; 128: 37-45.

4 Yu GP, Hsieh CC, Peng J. Risk factors associated with the prevalence of pulmonary tuberculosis among sanitary workers in Shanghai. Tubercle 1988; 69: 105-112.

5 Chang KC, Leung CC, Tam CM. Tuberculosis risk factors in a silicotic cohort in Hong Kong. Int J Tuberc Lung Dis 2001; 5: 177-184.

6 Kolappan C, Gopi PG. Tobacco smoking and pulmonary tuberculosis. Thorax 2002; 57: 964-966.

7 Leung CC, Yew WW, Chan CK, et al. Smoking and tuberculosis in Hong Kong. Int J Tuberc Lung Dis 2003; 7: 980-986.

8 Leung CC, Li T, Lam TH, et al. Smoking and tuberculosis among the elderly in Hong Kong. Am J Respir Crit Care Med 2004; 170: 1027-1033.

9 Doll R, Peto R, Wheatley K, Gray R, Sutherland I. Mortality in relation to smoking: 40 years' observations on male British doctors. BMJ 1994; 309: 901-911.

10 Liu BQ, Peto R, Chen ZM, et al. Emerging tobacco hazards in China: 1. Retrospective proportional mortality study of one million deaths. BMJ 1998; 317: 1411-1422.

11 Lam TH, Ho SY, Hedley AJ, Mak KH, Peto R. Mortality and smoking in Hong Kong: case-control study of all adult deaths in 1998. BMJ 2001; 323: 361.

12 Gajalakshmi V, Peto R, Kanaka TS, Jha P. Smoking and mortality from tuberculosis and other diseases in India: retrospective study of 43000 adult male deaths and 35000 controls. Lancet 2003; 362: 507-515.

13 Doll R, Hill AB. Lung cancer and other causes of mortality in relation to smoking; a second report on the mortality of British doctors. BMJ 1956; 12: 1071-1081.

14 Bothamley GH. Smoking and tuberculosis: a chance or causal association? Thorax 2005; 60: 527-528.

15 Tuberculosis and Chest Service. Annual report of Tuberculosis and Chest service (2004). Department of Health, Hong Kong, 2006.
16 Hong Kong Chest Service/Tuberculosis Research Centre, Madras/British Medical Research Council. A double-blind placebo-controlled clinical trial of three antituberculosis chemoprophylaxis regimens in patients with silicosis in Hong Kong. Am Rev Respir Dis 1992; 145: 36-41.

17 Levin ML. The occurrence of cancer in man. Acta Unio Int Contra Cancrum 1953; 19: 531.

18 Leung CC, Law WS, Chang KC, et al. Initial experience on rifampin and pyrazinamide versus isoniazid in the treatment of latent tuberculosis infection among patients with silicosis in Hong Kong. Chest 2003; 124: 2112-2118.

19 Hocking WG, Golde DW. The pulmonary-alveolar macrophage (first of two parts). N Engl J Med 1979; 301: 580-587.

20 Daniele RP, Dauber JH, Altose MD, Rowlands DT Jr, Gorenberg DJ. Lymphocyte studies in asymptomatic cigarette smokers. A comparison between lung and peripheral blood. Am Rev Respir Dis 1977; 116: 997-1005.

21 Wang H, Yu M, Ochani M, et al. Nicotinic acetylcholine receptor alpha7 subunit is an essential regulator of inflammation. Nature 2003; 421: 384-388.

22 Borovikova LV, Ivanova S, Zhang M, et al. Vagus nerve stimulation attenuates the systemic inflammatory response to endotoxin. Nature 2000; 405: 458-462.

23 Keane J, Gershon S, Wise RP, et al. Tuberculosis associated with infliximab, a tumor necrosis factor alpha-neutralizing agent. N Engl J Med 2001; 345: 1098-1104.

24 Lalvani A, Pathan AA, Durkan H, et al. Enhanced contact tracing and spatial tracking of Mycobacterium tuberculosis infection by enumeration of antigen-specific T cells. Lancet 2001; 357: 2017-2021.

25 Tuberculosis and Chest Service. Annual report of Tuberculosis and Chest service (1952). Department of Health, Hong Kong, 1953.

26 Brown KE, Campbell AH. Tobacco, alcohol and tuberculosis. Br J Dis Chest 1961; 55: 150-158.

27 Lewis JG, Chamberlain DA. Alcohol consumption and smoking habits in male patients with pulmonary tuberculosis. Br J Prev Soc Med 1963; 17: 149-152.

28 Leung CC, Chang KC, Law WS, et al. Determinants of spirometric abnormalities among silicotic patients in Hong Kong. Occup Med (Lond) 2005; 55: 490-493.

29 Chan-Yeung M, Tam CM, Wong H, et al. Molecular and conventional epidemiology of tuberculosis in Hong Kong: a population-based prospective study. J Clin Microbiol 2003; 41: 2706-2708. 